Agron. Mesoam. 29(1):19-28. Enero-abril, 2018

ISSN 2215-3608, doi:10.15517/ma.v29i1.27511

http://www.revistas.ucr.ac.cr/index.php/agromeso

\title{
Variabilidad patogénica de Colletotrichum lindemuthianum y resistencia en germoplasma de Phaseolus vulgaris L. de Ecuador ${ }^{1}$
}

\section{Pathogenic variability of Colletotrichum lindemuthianum and Ecuadorian Phaseolus vulgaris $\mathrm{L}$. germplasm resistance}

\author{
Diego Rodríguez-Ortega², Laura Vega-Jiménez², Ángel Murillo-Ilbay², Eduardo Peralta-Idrovo³, \\ Juan Carlos Rosas-Sotomayor ${ }^{4}$
}

\begin{abstract}
Resumen
La antracnosis causada por Colletotrichum lindemuthianum, es una de las enfermedades de mayor importancia económica del cultivo de fréjol (Phaseolus vulgaris L.) en Ecuador. La mejor alternativa de control es el uso de variedades resistentes. $C$. lindemuthianum presenta gran variabilidad patogénica lo que dificulta el desarrollo de variedades con resistencia duradera, por tanto, el conocimiento de la presencia y distribución de las razas fisiológicas del patógeno y la identificación de genes de resistencia son claves para desarrollar variedades con resistencia amplia y duradera. El objetivo de esta investigación fue determinar la variabilidad patogénica de $C$. lindemuthianum y la resistencia de genotipos de fréjol de Ecuador. La investigación se desarrolló en los años 2013 y 2014. Se caracterizaron diecisiete aislamientos de C. lindemuthianum procedentes de la región centro norte de Ecuador, por medio de la inoculación de un grupo de doce variedades diferenciales estándar. Entre las muestras analizadas se identificaron trece razas, cinco de las cuales no habían sido reportadas previamente en el país. La variedad diferencial G2333 (Co-4², Co-52 y Co-7) presentó resistencia a todas las razas caracterizadas en Ecuador. Adicionalmente, veintiún variedades mejoradas y líneas élite de fréjol fueron evaluadas con dieciséis de los diecisiete aislamientos caracterizados, se identificaron tres genotipos (TB2, TB3 e INIAP 485 Urcuquí), con resistencia a los aislamientos en mención. Las fuentes de resistencia identificadas en este estudio permitirán planificar el desarrollo de variedades de fréjol con resistencia amplia y duradera a C.lindemuthianum.
\end{abstract}

Palabras clave: antracnosis, razas fisiológicas, resistencia genética, variabilidad genética, enfermedades fungosas.

\begin{abstract}
Anthracnose caused by Colletotrichum lindemuthianum is one of the most economically important diseases of bean (Phaseolus vulgaris L.) cultivation in Ecuador. The best control alternative is the use of resistant varieties. C. lindemuthianum presents great pathogenic variability, which hinders the development of varieties with a lasting resistance, therefore, the knowledge of the presence and distribution of the physiological races of the pathogen and the
\end{abstract}

1 Recibido: 18 de enero, 2017. Aceptado: 13 de marzo, 2017. Este trabajo formó parte del proyecto "Incremento de la productividad de leguminosas (fréjol y arveja) mediante investigación en mejoramiento genético, sistemas de semillas y capacitación" en alianza estratégica entre el Instituto Nacional de Investigaciones Agropecuarias (INIAP) y la Secretaría de Educación Superior, Ciencia, Tecnología e Innovación (SENESCYT).

2 Instituto Nacional de Investigaciones Agropecuarias (INIAP). Programa Nacional de Leguminosas y Granos Andinos. Apartado postal 17-01-340. Quito, Ecuador. diego.rodriguez@iniap.gob.ec, laura.vega@iniap.gob.ec,angel.murillo@iniap.gob.ec

3 Exinvestigador del INIAP. peraltaedu55@gmail.com

4 Escuela Agrícola Panamericana Zamorano. Tegucigalpa, Honduras. Apartado aéreo No. 93. jcrosas@zamorano.edu 
identification of resistance genes are key to developing varieties with broad and lasting resistance. The objective of this research was to determine the pathogenic variability of $C$. lindemuthianum and to evaluate the resistance of Ecuadorian bean germplasm. The research was carried out between 2013 and 2014. Seventeen isolates of C. lindemuthianum from northern central Ecuador were characterized by the inoculation of a group of twelve standard differential bean varieties. Among the analyzed samples, thirteen races were identified; five of those races had not been previously reported in the country. The differential $\mathrm{G} 2333\left(\mathrm{Co}-4^{2}, \mathrm{Co}-5^{2}\right.$ and $\mathrm{Co}$-7) presented resistance to every characterized races in Ecuador. In addition, twenty - one improved varieties and elite bean lines were evaluated with sixteen of the seventeen isolates, three genotypes were identified (TB2, TB3 and INIAP 485 Urcuquí) with resistance to the mentioned isolates, which can be used as sources of resistance to Anthracnose. The identified sources of resistance in this study will allow to plan the development of bean varieties with broad and durable resistance to $C$. lindemuthianum.

Keywords: anthracnose, physiological races, genetic resistance, genetic variation, fungal diseases.

\section{Introducción}

En Ecuador, el fréjol común (Phaseolus vulgaris L.) es muy importante para la seguridad y soberanía alimentaria de la población, por su aporte de proteínas, carbohidratos y minerales (Peralta et al., 2007). Esta especie es cultivada en ambientes muy diversos, por lo que, es afectada por diversas enfermedades. Entre estas, la antracnosis ocasionada por el hongo Colletotrichum lindemuthianum aparece con mayor frecuencia en zonas ubicadas en altitudes superiores a $1500 \mathrm{msnm}$, con temperaturas entre 13 y $26^{\circ} \mathrm{C}$ y alta humedad relativa (entre 92 y 100\%) (Meneses et al., 1996; León, 2009).

La antracnosis puede causar daños de hasta el $90 \%$ en el rendimiento de granos en variedades susceptibles cultivadas en presencia del patógeno y bajo condiciones ambientales favorables para el desarrollo del mismo (Pastor-Corrales et al., 1994). La mejor alternativa de control de esta enfermedad es el uso de variedades resistentes, por las ventajas económicas, ambientales y sostenibles que presentan.

Para generar germoplasma resistente a $C$. lindemuthianum, es necesario disponer de suficiente información sobre la presencia y distribución de los patotipos de esta especie en las áreas de producción (González et al., 1998; Pinto et al., 2012). Una vez conocido el espectro de virulencia de las distintas razas del patógeno en la región, se puede recomendar la combinación de genes de resistencia más adecuada para incrementar la durabilidad y eficiencia de la resistencia a la antracnosis (Young et al., 1998; Awale et al., 2008).

La variabilidad patogénica de $C$. lindemuthianum se ha estudiado internacionalmente mediante el uso de doce variedades diferenciales estándar propuestas por el Centro Internacional de Agricultura Tropical (CIAT) (PastorCorrales, 1991). De estas doce variedades diferenciales cuatro son de origen andino y ocho mesoamericano (PastorCorrales, 1991). Los aislamientos de C. lindemuthianum provenientes de diferentes regiones son virulentos tanto en genotipos diferenciales de origen andino como mesoamericano (Menezes y Dianese, 1988; Balardin et al., 1997; Mahuku y Riascos, 2004).

En Ecuador, desde el 2003 hasta el 2010, con base en el grupo de doce genotipos diferenciales estándar (Santana y Mahuku, 2002), se han caracterizado 35 razas diferentes de C. lindemuthianum (Falconi et al., 2003; Mahuku y Riascos, 2004; INIAP, 2007; Nayibe et al., 2007; Awale et al., 2008; INIAP, 2010). En cada uno de estos estudios se reportó la presencia de nuevas razas. Adicionalmente, a pesar de que, en el país durante los últimos años se han generado líneas y variedades mejoradas de fréjol con resistencia a varias razas de antracnosis ya existentes, muchos de estos cultivares mejorados no han sido expuestos a la variabilidad de razas del patógeno que han sido caracterizadas; por lo que, es importante conocer y actualizar la variabilidad del patógeno y verificar el nivel de resistencia presente en estos genotipos. El objetivo de esta investigación fue determinar la variabilidad patogénica de $C$. lindemuthianum y la resistencia de genotipos de fréjol de Ecuador. 


\section{Materiales y métodos}

\section{Aislamiento y caracterización de razas de Colletotrichum lindemuthianum}

Durante los años 2013 y 2014 se colectaron diecisiete muestras de hojas, tallos y vainas infectadas naturalmente con antracnosis en campos de producción de fréjol de doce localidades, distribuidas en tres provincias (Imbabura, Pichincha y Chimborazo) de Ecuador. El número de muestras tomadas se definió con base en la importancia de la enfermedad en cada localidad.

Las muestras de hojas, tallos y vainas infectadas con antracnosis fueron trasladadas al laboratorio del Programa Nacional de Leguminosas y Granos Andinos (PRONALEG-GA), de la Estación Experimental Santa Catalina del Instituto Nacional de Investigaciones Agropecuarias (INIAP), e inmediatamente se procedió a aislar el hongo en medio de cultivo Mathur (Awale et al., 2008). Los aislamientos fueron incubados a $22{ }^{\circ} \mathrm{C}$ hasta su esporulación, seguido de tres cultivos monoconidiales y posteriormente, el incremento del inóculo.

Para el incremento del inóculo se añadió $10 \mathrm{ml}$ de agua destilada estéril a cada plato Petri que contenía el cultivo monoconidial esporulado, y se raspó la superficie del medio para obtener una suspensión de conidios, la cual se filtró a través de una doble capa de gasa para extraer la masa del micelio y restos de medio de cultivo. La concentración conidial se ajustó a 1,2 x $10^{6}$ conidias/ml, con el empleo de un hematocitómetro (Awale et al., 2008; Ferreira et al., 2008; Vega, 2011).

Para la identificación de las razas de antracnosis se utilizaron las doce variedades diferenciales estándar de fréjol común, descritas por Pastor-Corrales (1991): Michelite (Co-11), Michigan Dark Red Kidney (Co-1), Perry Marrow $\left(\mathrm{Co}-\mathrm{l}^{3}\right)$, Cornell $49242(\mathrm{Co}-2)$, Widusa $\left(\mathrm{Co}-\mathrm{I}^{5}\right)$, Kaboon $\left(\mathrm{Co}-\mathrm{l}^{2}\right)$, México $222(\mathrm{Co}-3)$, PI $207262\left(\mathrm{Co}-4^{3}\right.$, Co-9), TO (Co-4), TU (Co-5), AB 136 (Co-6) y G 2333 (Co-42, $\left.\mathrm{Co}-5^{2}, \mathrm{Co}-7\right)$.

Ocho semillas de cada variedad diferencial fueron sembradas en dos bandejas plásticas (cuatro semillas por bandeja). Quince días después de la siembra las plántulas fueron inoculadas por aspersión sobre las hojas primarias, tanto en el haz como en el envés. Después que las plántulas permanecieron en una cámara húmeda ( $>95 \%$ humedad relativa) durante 48 horas, fueron transferidas a un invernadero con una temperatura promedio de $18{ }^{\circ} \mathrm{C} \pm 3{ }^{\circ} \mathrm{C}$ hasta su evaluación (González et al., 2004; Awale et al., 2008). La temperatura y humedad ambiental del sitio del ensayo fueron registradas con un termohigrómetro.

La reacción de las variedades diferenciales fue registrada a los doce días después de la inoculación de acuerdo con la escala descriptiva de 1 al 9 del CIAT (1987), en donde un valor de 1 indica plantas sin síntomas visibles de la enfermedad y un valor de 9 indica plantas severamente enfermas o muertas. Reacciones con valoración de 1 al 3 se consideran resistentes (reacción incompatible, representada por el signo -), mientras que reacciones con puntajes 4 al 9 son consideradas susceptibles (reacción compatible, representada con el signo +) (Balardin et al., 1997; Del-Río et al., 2003).

\section{Evaluación de germoplasma mejorado de fréjol}

Posterior a la caracterización de los aislamientos de C. lindemuthianum, plántulas de veintidós genotipos de fréjol (trece variedades mejoradas, siete líneas élite y dos controles positivos: Paragachi y Cocacho), fueron inoculados con dieciséis de los diecisiete aislamientos utilizados en el estudio de variabilidad patogénica de la presente investigación. Para el manejo del patógeno, inoculación y evaluación se utilizó la metodología previamente descrita. 


\section{Resultados}

\section{Caracterización de razas}

Diferentes aislamientos, colectados en tres provincias productoras de fréjol de la Sierra Ecuatoriana, se caracterizaron en función a la reacción de los doce diferenciales. En los diecisiete aislamientos estudiados se identificaron trece razas: nueve en la provincia de Imbabura y dos en cada una de las provincias de Pichincha y Chimborazo (Cuadro 1). Cinco de estas razas $(39,263,295,311$ y 453) no han sido reportadas anteriormente en Ecuador.

Cuadro 1. Caracterización de aislamientos de C. lindemuthianum colectados en las provincias de Imbabura, Pichincha y Chimborazo en Ecuador. 2014.

Table 1. Characterization of collected isolates of Colletotrichum lindemuthianum found in Imbabura, Pichincha and Chimborazo provinces in Ecuador. 2014.

\begin{tabular}{|c|c|c|c|c|c|c|c|c|c|c|c|c|c|c|}
\hline \multicolumn{2}{|c|}{ Procedencia aislamientos } & \multicolumn{12}{|c|}{ Genotipos diferenciales } & \multirow{3}{*}{ Raza } \\
\hline \multirow[t]{2}{*}{ Provincia } & \multirow[t]{2}{*}{ Localidad } & 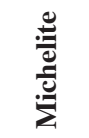 & 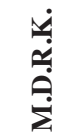 & 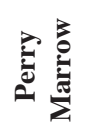 & 焉 & $\underset{3}{\stackrel{g}{\varrho}}$ & 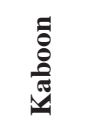 & 䓤 సี & 총 & $\stackrel{0}{=}$ & ? & 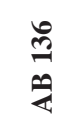 & లై & \\
\hline & & Co-11 & Co-1 & $\operatorname{Co} 1^{3}$ & Co 2 & Co $1^{5}$ & $\operatorname{Co} 1^{2}$ & Co 3 & $\begin{array}{c}\operatorname{Co~} 4^{3}, \\
\operatorname{Co} 9 \\
\end{array}$ & Co 4 & Co 5 & Co 6 & $\begin{array}{c}\mathrm{Co}^{2}, \mathrm{Co} \\
5^{2}, \mathrm{Co} 7 \\
\end{array}$ & \\
\hline \multirow{13}{*}{ Imbabura } & \multirow{4}{*}{ Urcuquí } & + & + & - & - & - & - & - & + & + & - & - & - & 387 \\
\hline & & + & - & + & - & - & - & - & + & + & - & - & - & 389 \\
\hline & & + & - & + & - & - & - & - & + & + & - & - & - & 389 \\
\hline & & + & + & + & - & - & - & - & - & + & - & - & - & 263 \\
\hline & Pablo Arenas & + & + & + & - & - & - & - & + & + & - & - & - & 391 \\
\hline & San Rafael & + & - & + & - & - & - & - & + & + & - & - & - & 389 \\
\hline & Quiroga & - & - & - & - & - & - & - & - & + & - & - & - & 256 \\
\hline & Otavalo & + & - & + & - & - & - & - & - & - & - & - & - & 5 \\
\hline & Carpuela & - & - & + & - & - & - & - & - & - & - & - & - & 4 \\
\hline & Imantag & + & + & + & - & - & - & - & + & + & - & - & - & 391 \\
\hline & San Pablo & + & - & + & - & - & - & - & - & - & - & - & - & 5 \\
\hline & Cahuasquí & + & - & + & - & - & - & + & + & + & - & - & - & 453 \\
\hline & Caldera & - & - & + & - & - & - & - & - & + & - & - & - & 260 \\
\hline \multirow{2}{*}{ Pichincha } & \multirow{2}{*}{ Tumbaco } & + & + & + & - & + & + & - & - & + & - & - & - & 311 \\
\hline & & + & - & + & - & - & + & - & - & + & - & - & - & 293 \\
\hline \multirow{2}{*}{ Chimborazo } & \multirow{2}{*}{ Pallatanga } & + & + & + & - & - & + & - & - & - & - & - & - & 39 \\
\hline & & + & + & + & - & - & + & - & - & + & - & - & - & 295 \\
\hline
\end{tabular}

Reacción susceptible (+) y resistente (-) a la inoculación con los aislamientos de C. lindemuthianum Ecuador / Susceptible (+) and resistant (-) reaction to inoculation of C. lindemuthianum isolates from Ecuador.

La raza 311 fue la más virulenta, ya que produjo reacciones de susceptibilidad en seis de las doce variedades diferenciales de fréjol, seguida por 295, 391 y 453. Las razas menos virulentas fueron 4 y 256.

La variedad diferencial Perry Marrow $\left(\mathrm{Co}^{-1} \mathrm{l}^{3}\right)$ presentó susceptibilidad a doce de los diecisiete aislamientos, seguida por Michelite (Co-11) y TO (Co-4). 
Las variedades diferenciales Cornell 49242 (Co-2), TU (Co-5), AB 136 (Co-6) y G2333 (Co-42 Co-5 $^{2}$ y Co-7) presentaron resistencia para los diecisiete aislamientos de C. lindemuthianum.

\section{Evaluación de germoplasma mejorado de fréjol}

De los veintidós genotipos de fréjol evaluados en este estudio, las líneas promisorias TB2 y TB3 y la variedad INIAP 485 Urcuquí, presentaron resistencia a los dieciséis aislamientos de $C$. lindemuthianum. Otros cultivares como INIAP 484 Centenario, INIAP 414 Yunguilla y AMPR5 fueron resistentes a diez aislamientos (Cuadro 2). Se identificaron genotipos de fréjol que presentaron diferente respuesta de resistencia y susceptibilidad a aislamientos procedentes de diferentes localidades, pero caracterizados como la misma raza (Cuadro 2). Esto sucedió con las razas: 5, 389 y 391, que fueron las que se identificaron en dos o más localidades.

Cuadro 2. Reacción de genotipos de fréjol común a dieciséis aislamientos de Colletotrichum lindemuthianum de Ecuador. 2014. Table 2. Reaction of common bean genotypes to sixteen isolates of Ecuadorian Colletotrichum lindemuthianum. 2014.

\begin{tabular}{|c|c|c|c|c|c|c|c|c|c|c|c|c|c|c|c|c|}
\hline \multirow{3}{*}{ Genotipos/razas } & \multicolumn{16}{|c|}{ Origen de aislamientos y razas caracterizadas } \\
\hline & نَّ & 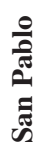 & 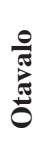 & : & $\frac{\pi}{0}$ & 氕 & $\begin{array}{l}\stackrel{\Xi}{\Xi} \\
\text { E } \\
\text { E }\end{array}$ & 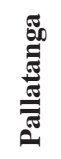 & 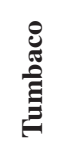 & 营 & 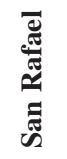 & 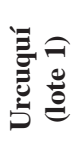 & 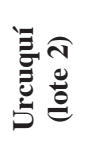 & 율 & & 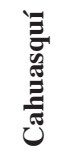 \\
\hline & 4 & 5 & 5 & 256 & 260 & 263 & 293 & 295 & 311 & 387 & 389 & 389 & 389 & 391 & 391 & 453 \\
\hline Paragachi (testigo) & + & + & + & + & + & + & + & + & + & + & + & + & + & + & + & + \\
\hline INIAP 414 Yunguilla & - & - & - & - & + & - & - & - & - & + & - & - & - & + & - & - \\
\hline INIAP 484 Centenario & + & - & - & - & - & - & - & + & + & - & - & - & - & - & - & - \\
\hline $\begin{array}{l}\text { INIAP } 483 \text { Intag } \\
\text { INIAP } 424\end{array}$ & + & + & + & - & + & + & + & + & + & + & - & - & + & - & - & + \\
\hline Concepción & + & + & + & - & + & + & + & + & + & + & - & + & + & + & + & + \\
\hline INIAP 482 Afroandino & - & - & - & - & + & + & - & - & - & + & - & + & + & + & + & + \\
\hline INIAP 429 Paragachi & & & & & & & & & & & & & & & & \\
\hline Andino & - & - & - & + & - & - & - & - & + & - & + & - & + & - & - & + \\
\hline INIAP 425 Fanesquero & + & - & - & - & + & + & + & + & + & - & + & - & + & + & + & + \\
\hline INIAP 485 Urcuqui & - & - & - & - & - & - & - & - & - & - & - & - & - & - & - & - \\
\hline INIAP 480 Rocha & + & - & - & - & + & + & + & + & + & + & + & + & + & - & + & + \\
\hline INIAP 422 Blanco & & & & & & & & & & & & & & & & \\
\hline Belén & - & - & - & - & - & - & + & + & - & + & - & + & - & + & + & + \\
\hline INIAP 420 Chota & + & - & - & - & + & + & + & + & + & + & + & + & + & + & + & + \\
\hline INIAP 427 Libertador & + & - & - & + & - & + & + & - & + & - & - & - & - & - & - & - \\
\hline INIAP 428 Canario & & & & & & & & & & & & & & & & \\
\hline Guarandeño & + & + & + & - & + & - & + & + & + & + & + & + & + & + & + & + \\
\hline AMPR 5 & - & - & - & - & - & - & - & + & + & - & - & - & - & - & - & - \\
\hline CAL 143 & + & + & + & + & + & + & - & - & - & + & + & - & - & + & + & - \\
\hline G 916 & + & + & - & + & - & - & + & - & - & - & - & + & + & + & + & + \\
\hline TB 3 & - & - & - & - & - & - & - & - & - & - & - & - & - & - & - & - \\
\hline TB 2 & - & - & - & - & - & - & - & - & - & - & - & - & - & - & - & - \\
\hline TRM 1 & - & + & + & - & - & + & - & - & - & - & - & - & - & - & - & - \\
\hline POA 10 & + & - & - & - & + & - & + & + & + & + & - & - & - & - & - & - \\
\hline $\begin{array}{l}\text { Cocacho (testigo } \\
\text { susceptible) }\end{array}$ & + & + & + & + & + & + & + & + & + & + & + & + & + & + & + & + \\
\hline
\end{tabular}

Reacción susceptible (+) y resistente (-) a la inoculación con los aislamientos de C. lindemuthianum Ecuador / Susceptible (+) and resistant (-) reaction to inoculation with Ecuadorian isolates of $C$. lindemuthianum. 
Como resultado de la reacción de resistencia-susceptibilidad de los veintidós genotipos de fréjol, a los dieciséis aislamientos de $C$. lindemuthianum, se determinó que las razas 4 y 311 (caracterizadas con las variedades diferenciales), fueron las más virulentas, por infectar trece líneas/variedades mejoradas de fréjol. En contraste, la raza 256 fue la menos virulenta, infectó únicamente a seis líneas/variedades mejoradas (Cuadro 2).

\section{Discusión}

C. lindemuthianum es conocido mundialmente por presentar una amplia variabilidad patogénica (Kelly et al., 1994; Araya, 2003; Rodríguez-Guerra et al., 2006; Sánchez-García et al., 2009; Campa et al., 2011; Pinto et al., 2012) y los resultados obtenidos en este estudio lo confirmaron al identificar trece razas diferentes de diecisiete aislamientos provenientes de tres provincias de Ecuador.

Los principales mecanismos por los que C. lindemuthianum genera variabilidad genética son las mutaciones, flujo de genes entre poblaciones y recombinación genética (Gallego et al., 2010). Por lo que, se debe realizar un monitoreo constante tanto de las poblaciones del patógeno, así como de los genes de resistencia (Gallego et al., 2010; Pinto et al., 2012).

De las trece razas identificadas, cinco son reportadas por primera vez en Ecuador; estas cinco razas $(39,263$, 295, 311 y 453) provienen de aislamientos colectados en localidades que no han sido muestreadas en estudios previos, a excepción de la raza 311 que fue caracterizada en un aislamiento procedente de la localidad de Tumbaco (Pichincha), lugar en el que se han identificado las razas 1031 (Awale et al., 2008), 813 y 941 (INIAP, 2010).

Las poblaciones del patógeno de origen andino son más virulentas con las variedades diferenciales del acervo andino, debido a la mayor adaptación de razas a cultivares de la misma región geográfica (coevolución hospedante-patógeno) (Balardin et al., 1997; González et al., 1998; Araya, 2003; Gonçalves-Vidigal et al., 2012). De manera general, esto fue observado en los resultados de este estudio, ya que las variedades diferenciales de origen mesoamericano con sus respectivos genes: Cornell 49242 (Co-2), TU (Co-5), AB 136 (Co-6), G2333 $\left(\mathrm{Co}-4^{2}, \mathrm{Co}-5^{2}, \mathrm{Co}-7\right)$, presentaron resistencia para todos los aislamientos analizados. Sin embargo, también se observaron excepciones, ya que la diferencial Michellite (Co-11), a pesar de ser de origen mesoamericano, presentó susceptibilidad a la mayoría de aislamientos. Es importante mencionar que la raza 453 (identificada por primera vez en este estudio) fue la única que indujo respuesta de susceptibilidad en la variedad diferencial mesoamericana México 222 (Co-3). Esta diferencial ha sido reportada como resistente en estudios previos por lo que, esta raza rompe la resistencia conferida por el gen Co-3 (Falconi et al., 2003; Mahuku y Riascos, 2004; INIAP, 2007; Awale et al., 2008; INIAP, 2010; Vega, 2011).

Las variedades diferenciales de origen andino: MDRK (Co-1) y Perry Marrow $\left(\mathrm{Co}-\mathrm{l}^{3}\right)$ presentaron

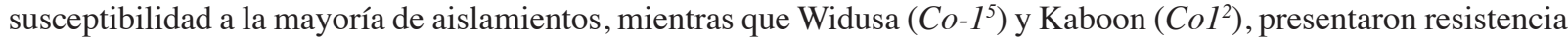
a la mayoría de aislamientos. Estos resultados indican que en Ecuador los aislamientos de C. lindemuthianun considerados de origen andino (ya que se siembran variedades de fréjol principalmente de origen andino), no necesariamente inducirán reacciones de susceptibilidad en todos los genotipos de fréjol andino y reacciones de resistencia en todos los genotipos mesoamericanos. Resultados similares han sido obtenidos en diferentes países (Menezes y Dianese, 1988; Balardin et al., 1997; Mahuku y Riascos, 2004).

La principal vía de diseminación (a grandes distancias) de la antracnosis es a través de la semilla, por lo que, no se descarta que las razas identificadas en localidades específicas puedan posteriormente ser introducidas en localidades en las que no se encontraban. Por esta razón, es importante revisar y tomar en cuenta todas las razas de C. lindemuthianum identificadas en diferentes estudios realizados a través del tiempo en Ecuador.

En estudios previos sobre la variabilidad patogénica de $C$. Indemuthianum en Ecuador, se observa que se han identificado un total de 35 razas diferentes (Cuadro 3) (Falconi, et al., 2003; Mahuku y Riascos, 2004; INIAP, 
Rodríguez-Ortega et al.: Variabilidad patogénica de la antracnosis de fréjol

Cuadro 3. Razas de Colletotrichum lindemuthianum identificadas en Ecuador en estudios previos.

Table 3. Ecuadorian characterized Colletotrichum lindemuthianum races in previous studies.

\begin{tabular}{ccccccccccc}
\hline \multicolumn{10}{c}{ Razas caracterizadas } & Fuente \\
\hline 0 & 3 & 4 & 256 & 260 & 1346 & & & & Falconi et al. (2003) \\
0 & 1 & 4 & 5 & 6 & 7 & 9 & 15 & 65 & 128 & Mahuku y Riascos (2004) \\
129 & 133 & 256 & 261 & 1153 & & & & & & \\
0 & 4 & 7 & 256 & 260 & 261 & 321 & 448 & 647 & & INIAP (2007) \\
4 & 7 & 12 & 13 & 1031 & & & & & & Awale et al. (2008) \\
293 & 301 & 429 & 813 & 941 & & & & & & INIAP (2010) \\
0 & 256 & 261 & 293 & 309 & 387 & 389 & 391 & 1280 & 1284 & Vega (2011) \\
\hline
\end{tabular}

2007; Awale et al., 2008; INIAP, 2010; Vega, 2011). Al añadir las cinco razas identificadas en el presente estudio (reportadas por primer vez) se tendría un total de cuarenta razas de $C$ lindemuthianum en Ecuador. Adicionalmente al revisar los estudios mencionados, la combinación de las cuarenta razas inducen respuestas de susceptibilidad sobre once de las doce variedades diferenciales estándar. G2333, que posee los genes $\operatorname{Co}^{2}, \operatorname{Co}^{2}$ y $C o 7$, es la única variedad diferencial que presentó resistencia.

En referencia a los genes presentes en la variedad diferencial G2333, se desconoce si el efecto individual de uno de los tres genes, o las diferentes combinaciones de los mismos, están confiriendo la resistencia a todas las razas del patógeno identificadas. No obstante, lo más probable es que la combinación de al menos dos genes son los que confieren la resistencia a todas las razas de Ecuador, ya que según de-Freitas y Stadnik (2012), no se han reportado genes $C o$ individuales que confieran resistencia a todas las razas conocidas de $C$. lindemuthianum.

Con respecto a la evaluación del germoplasma mejorado de fréjol, según los resultados se observa que todos estos genotipos poseen genes de resistencia para algunos de los aislamientos evaluados. Varios de estos genes inducen un amplio rango de resistencia e inclusive los presentes en las líneas TB2 y TB3, así como en la variedad INIAP 485 Urcuquí, provocaron reacciones de resistencia a todas las razas de $C$. lindemuthianum evaluadas en este estudio.

Se observó que aislamientos procedentes de diferentes localidades y caracterizados como la misma raza presentaron diferentes respuestas de resistencia y susceptibilidad en el mismo genotipo mejorado de fréjol. De manera general, las variedades de los cultivos poseen ciertos genes de resistencia o susceptibilidad que reaccionan en forma diferente contra varias razas del patógeno y sus genes de virulencia o avirulencia (Castaño, 1994). Considerando la gran variabilidad genética, tanto del patógeno como del fréjol común, el uso de las doce variedades diferenciales (de las cuales solamente cuatro son de origen andino), no son suficientes para explicar la gran variabilidad en este patosistema. Resultados similares han sido publicados por Falconi et al. (2003).

Los genotipos, en los que se observó diferente reacción de resistencia-susceptibilidad a la misma raza de este hongo, permiten una mejor explicación de la interacción patógeno-hospedante a nivel local. Un ejemplo de lo mencionado es que en este estudio con el uso de las variedades diferenciales la raza 4 fue la menos virulenta, mientras que esta misma raza indujo reacciones de susceptibilidad en la mayoría de los genotipos mejorados de fréjol evaluados, resultando como la raza más virulenta. Por esta razón, es de suma importancia incluir genotipos locales (a más de las variedades diferenciales) durante estudios de caracterización patogénica de C. lindemuthianum.

Una estrategia para incrementar la durabilidad y eficiencia de la resistencia a $C$. lindemuthianum es la piramidación de varios genes en los cultivares de fréjol, una vez que el espectro de virulencia de las distintas razas del hongo en la región sea conocido, para así recomendar la combinación de genes de resistencia más adecuada (Young et al., 1998). Según los resultados obtenidos en este estudio y tomando en cuenta estudios previos (Falconi 
et al., 2003; Mahuku y Riascos, 2004; INIAP, 2007; Awale et al., 2008; INIAP, 2010; Vega, 2011), la piramidación de los genes presentes en la variedad diferencial G233 (Co-42, $\mathrm{Co}-5^{2}$ y $\left.\mathrm{Co}-7\right)$, en la variedad mejorada INIAP 485 Urcuquí y en las líneas TB 2 y TB 3, proporcionarán resistencia a todas las razas de $C$. lindemuthianum identificadas hasta hoy en Ecuador. Es importante destacar que el o los genes de resistencia presentes en los tres genotipos mejorados de fréjol mencionados son desconocidos. Se han identificado veinte genes que inducen reacciones de resistencia a varias razas o aislamientos de $C$. lindemuthianum (Ferreira et al., 2013). Sería muy importante realizar futuros estudios genéticos para identificar si los genes presentes en los tres genotipos resistentes de fréjol mencionados, ya han sido reportados o se trata de genes distintos.

\section{Conclusiones}

Hay gran variabilidad patogénica de $C$. lindemuthianum en Ecuador, debido a la identificación de trece razas, de las cuales las 39, 263, 295, 311 y 453 son reportadas por primera vez.

De veintidós genotipos mejorados de fréjol, las líneas élite TB2, TB3, y la variedad INIAP 485 Urcuquí fueron resistentes a todos los aislamientos evaluados. Por lo cual, la piramidación de los genes de resistencia (en diferentes combinaciones) presentes en estos genotipos, son recomendados para generar variedades mejoradas con resistencia duradera a la antracnosis en Ecuador.

\section{Literatura citada}

Araya, C.M. 2003. Coevolución de interacciones hospedante - patógeno en frijol común. Fitopatol. Bras. 28:221-228.

Awale, H., E. Falconí-Castillo, J. Villatoro-Mérida, y J. Kelly. 2008. Caracterización de aislamientos de Colletotrichum lindemuthianum de Ecuador y Guatemala para identificar genes de resistencia. Agron. Mesoam. 19:1-6. doi:https://doi. org/10.15517/am.v19i1.5016

Balardin, R., A. Jarosz, and J.D. Kelly. 1997. Virulence and molecular diversity in Colletotrichum lindemuthianum from South, Central, and North America. Phytopathol. 87:1184-1191. doi:10.1094/PHYTO.1997.87.12.1184

Castaño, J. 1994. Principios básicos de fitopatología. $2^{\text {da }}$ ed. Zamorano Academic Press, Zamorano, HON.

Campa, A., R. Giraldez, and J.J. Ferreira. 2011. Genetic analysis of the resistance to eight anthracnose races in the common bean differential cultivar Kaboon. Phytopathol. 101:757-764. doi:10.1094/PHYTO-11-10-0296

CIAT. 1987. Sistema estándar para la evaluación de germoplasma de frijol. CIAT, Cali, COL.

de-Freitas, MB, and M.J. Stadnik. 2012. Race-specific and ulvan-induced defense responses in bean (Phaseolus vulgaris) against Colletotrichum lindemuthianum. Physiol. Mol. Plant Pathol. 78:8-13. doi:10.1016/j.pmpp.2011.12.004

Del-Río, L.E., R.S. Lamppa, and P.L. Gross. 2003. Characterization of the reaction of North Dakota dry bean cultivars to three races of Colletotrichum lindemuthianum. Plant Dis. 87:263-265. doi:10.1094/PDIS.2003.87.3.263

Falconi, E., J.B. Ochoa, E. Peralta, and D. Danial. 2003. Virulence patterns of Colletotrichum lindemuthianum in common bean in Ecuador. Annu. Rept. Bean Improv. Coop. 46:167-168.

Ferreira, J., A. Campa, and J. Kelly. 2013. Organization of genes conferring resistance to anthracnose in common bean. In: R.K. Varshney, and R. Tuberosa, editors, Translational genomic for crop breeding: biotic stress. John Wiley \& Sons, Chichester, GBR. p. 151-182. doi:10.1002/9781118728475.ch9

Ferreira, J.J., A. Campa, E. Pérez-Vega, and R. Girales. 2008. Reaction of a bean germplasm collection against five races of Colletotrichum lindemuthianum identified in northern Spain and implications for breeding. Plant Dis. 92:705-708. doi:10.1094/PDIS-92-5-0705

26

Agron. Mesoam. 29(1):19-28, enero-abril, 2018 ISSN 2215-3608 doi:10.15517/ma.v29i1.27511 
Gallego, C., G.A. Ligarreto, L.N. Garzón, O.A. Oliveros, y L.J. Rincón. 2010. Rendimiento y reacción a Colletotrichum lindemuathianum en cultivares de fríjol voluble (Phaseolus vulgaris L.). Rev. Fac. Nal. Agron. Medellín 63:5477-5488.

Gonçalves-Vidigal, M.C., A.C. Meirelles, J.P. Poletine, L.L. De Sousa, A.S. Cruz, M.P. Nunes, G.F. Lacanallo, and P.S. Vidigal-Filho. 2012: Genetic analysis of anthracnose resistance in Pitanga dry bean cultivar. Plant Breed. 131:423-429. doi:10.1111/j.1439-0523.2011.01939.x

González, M., R. Rodríguez, F. Hernández, J. Acosta, O. Martínez, and J. Simpson 2004. Analysis of pathotypes of Colletotrichum lindemuthianum found in the Central Region of Mexico and resistance in elite germplasm of Phaseolus vulgaris. Plant Dis. 88:152-156. doi:10.1094/PDIS.2004.88.2.152

González, M., R. Rodríguez, M.E. Zavala, J.L. Jacobo, F. Hernández, J. Acosta, O. Martínez, and J. Simpson. 1998. Characterization of Mexican isolates of Colletotrichum lindemuthianum by using differential cultivars and molecular markers. Phytopathol. 88:292-299. doi:10.1094/PHYTO.1998.88.4.292

INIAP (Instituto Nacional de Investigaciones Agropecuarias). 2007. Informe anual de actividades 2006. INIAP, Programa Nacional de Leguminosas y Granos Andinos, Estación Experimental Santa Catalina, Quito, ECU.

INIAP (Instituto Nacional de Investigaciones Agropecuarias). 2010. Informe anual de actividades 2009. INIAP, Programa Nacional de Leguminosas y Granos Andinos, Estación Experimental Santa Catalina, Quito, ECU.

Kelly, J.D., L. Afanador, and L.S. Cameron. 1994. New races of Colletotrichum lindemuthianum in Michigan and implications in dry bean resistance breeding. Plant Dis. 78:892-894.

León, I. 2009. La antracnosis y la mancha angular del fríjol común (Phaseolus vulgaris L.). Temas Cienc. Tecnol. 13(39):45-54.

Mahuku, G., and J. Riascos. 2004. Virulence and molecular diversity within Colletotrichum lindemuthianum isolates from Andean and Mesoamerican bean varieties and regions. European J. Plant Pathol. 110:253-263. doi:10.1023/ B:EJPP.0000019795.18984.74

Menezes, J., and J. Dianese. 1988. Race characterization of Brazilian isolates of Colletotrichum lindemuthianum and detection of resistance to anthracnose in Phaseolus vulgaris L. Phytopathol. 78:650-655.

Meneses, R., H. Waaijenberg, y L. Piérola. 1996. Las leguminosas en la agricultura boliviana: revisión de información. Impresores Colorgraf, Cochabamba, BOL.

Nayibe, L., M. Blair, y G. Ligarreto. 2007. Uso de selección asistida con marcadores para resistencia a antracnosis en fríjol común. Agron. Colomb. 25:207-214.

Pastor-Corrales, M.A. 1991. Estandarización de variedades diferenciales y designación de razas de Colletotrichum lindemuthianum. Phytopathol. 81:694.

Pastor-Corrales, M.A., O.A. Erazo, E.I. Estrada, and S.P. Singh. 1994. Inheritance of anthracnose resistance in common bean accession G 2333. Plant Dis. 78:959-962. doi:10.1094/PD-78-0959

Peralta, E., A. Murillo, E. Falconí, N. Mazón, y J. Pinzón. 2007. Manual de campo para el reconocimiento y control de las enfermedades más importantes que afectan al cultivo de fréjol (Phaseolus vulgaris L.) en Ecuador. Publicación Miscelánea No. 136. INIAP, Programa Nacional de Leguminosas y Granos Andinos, Estación Experimental Santa Catalina, Quito, ECU.

Pinto, J.M., R. Pereira, S.F. Mota, F.H. Ishikawa, and E.A. Souza. 2012. Investigating Phenotypic Variability in Colletotrichum lindemuthianum Populations. Phytopathol. 102:490-497.

Rodríguez-Guerra, R., J.A. Acosta-Gallegos, M.M. González-Chavira, y J. Simpson. 2006. Patotipos de Colletotrichum lindemuthianum y su implicación en la generación de cultivares resistentes de frijol. Agric. Téc. Méx. 32:101-114. 
Sánchez-García, B.M., A. Flores-Olivas, A. Sánchez-Arizpe, S. Pineda-Rodríguez, G. López-Jiménez, S. Fraire-Velásquez, E.R. Garrido-Ramírez, J.A. Acosta-Gallegos, J. Simpson-Williamson, y R. Rodríguez-Guerra. 2009. Patotipos de Colletotrichum lindemuthianum en Oaxaca y San Luis Potosí, México, y resistencia en genotipos de frijol. Agric. Téc. Méx. 35:46-57.

Santana, G.E., y G. Mahuku. 2002. Diversidad de razas de Colletotrichum lindemuthianum en Antioquia y evaluación de germoplasma de fréjol crema-rojo por resistencia a antracnosis. Agron. Mesoam. 13:95-103. doi:10.15517/am.v13i2.12024

Vega, L. 2011. Análisis de la resistencia de poblaciones locales de fréjol (Phaseolus vulgaris L.) de Cotacachi y Saraguro a antracnosis (Colletotrichum lindemuthianum) y roya (Uromyces appendiculatus). Tesis Ing. Agr. Universidad Central del Ecuador, Quito, ECU.

Young, R.A., M. Melotto, R.O. Nodari, and J.D. Kelly. 1998. Marker assisted dissection of oligogenic anthracnose resistance in the common bean cultivar G2333. Theor. Appl. Genet. 96:87-94. doi:10.1007/s001220050713 